\title{
Fractional Flow Reserve
}

Estimated at Coronary CT

Angiography in Intermediate

Lesions: Comparison of Diagnostic

Accuracy of Different Methods to

\section{Determine Coronary Flow Distribution ${ }^{1}$}

Satoru Kishi, MD

Andreas A. Giannopoulos, MD

Anji Tang, BS

Nahoko Kato, MD

Yiannis S. Chatzizisis, MD, PhD

Carole Dennie, MD

Yu Horiuchi, MD

Kengo Tanabe, MD, PhD

João A. C. Lima, MBA, MD

Frank J. Rybicki, MD, PhD

Dimitris Mitsouras, PhD
${ }^{1}$ From the Division of Diabetes, Mitsui Memorial Hospital, Tokyo, Japan (S.K.); Applied Imaging Science Laboratory, Department of Radiology, Brigham and Women's Hospital, Harvard Medical School, 75 Francis St, Boston, MA 02115 (A.A.G., A.T., D.M.); Division of Cardiology, Mitsui Memorial Hospital, Tokyo, Japan (N.K., Y.H., K.T.); Department of Cardiology, University of Nebraska Medical Center, Omaha, $\mathrm{Neb}$ (Y.S.C.); Department of Radiology, the University of Ottawa Faculty of Medicine, and the Ottawa Hospital Research Institute, Ottawa, Canada (C.D., F.J.R., D.M.); and Department of Cardiovascular Imaging, Johns Hopkins Medicine, Baltimore, Md (J.A.C.L.). Received November 22, 2016; revision requested January 23, 2017; revision received July 6; accepted August 1; final version accepted September 7. Address correspondence to D.M. (e-mail: dmitsouras@alum.mit.edu).

Supported by National Institute of Biomedical Imaging and Bioengineering (EB015868), Toshiba America Medical Systems.

S.K. and A.A.G. contributed equally to this work.

- RSNA, 2017
Purpose:

Materials and Methods:

Results:

Conclusion:
To compare the diagnostic accuracy of different computed tomographic (CT) fractional flow reserve (FFR) algorithms for vessels with intermediate stenosis.

This cross-sectional HIPAA-compliant and human research committee-approved study applied a four-step CT FFR algorithm in 61 patients (mean age, 69 years \pm 10 ; age range, 29-89 years) with a lesion of intermediate-diameter stenosis (25\%-69\%) at CT angiography who underwent FFR measurement within 90 days. The per-lesion diagnostic performance of CT FFR was tested for three different approaches to estimate blood flow distribution for CT FFR calculation. The first two, the Murray law and the Huo-Kassab rule, used coronary anatomy; the third used contrast material opacification gradients. CT FFR algorithms and CT angiography percentage diameter stenosis (DS) measurements were compared by using the area under the receiver operating characteristic curve (AUC) to detect FFRs of 0.8 or lower.

Twenty-five lesions (41\%) had FFRs of 0.8 or lower. The AUC of CT FFR determination by using contrast material gradients $(\mathrm{AUC}=0.953)$ was significantly higher than that of the Huo-Kassab (AUC $=0.882, P=.043$ ) and Murray law models $(\mathrm{AUC}=0.871, P=.033)$. All three AUCs were higher than that for $50 \%$ or greater DS at CT angiography $(\mathrm{AUC}=0.596, P<.001)$. Correlation of CT FFR with FFR was highest for gradients (Spearman $\rho=0.80$ ), followed by the Huo-Kassab rule $(\rho=0.68)$ and Murray law $(\rho=0.67)$ models. All CT FFR algorithms had small biases, ranging from -0.015 (Murray) to -0.049 (Huo-Kassab). Limits of agreement were narrowest for gradients $(-0.182,0.147)$, followed by the Huo-Kassab rule $(-0.246,0.149)$ and the Murray law $(-0.285,0.256)$ models.

Clinicians can perform CT FFR by using a four-step approach on site to accurately detect hemodynamically significant intermediate-stenosis lesions. Estimating blood flow distribution by using coronary contrast opacification variations may improve CT FFR accuracy.

${ }^{\circ}$ RSNA, 2017

Online supplemental material is available for this article. 
C oronary fractional flow reserve (FFR) is the reference standard measurement for directing appropriate percutaneous coronary intervention (1). Computed tomographic (CT) FFR technology that noninvasively estimates FFR from CT angiography data by using computational fluid dynamics (CFDs) may deliver cost savings and improved quality of life (2). To date, three CT FFR technologies have been validated against conventional FFR in the peer-reviewed literature (3-8).

\section{Advances in Knowledge}

- The diagnostic accuracy of CT fractional flow reserve (FFR) estimation in revealing invasive FFR of 0.8 or less in intermediate lesions $(25 \%-69 \%$ diameter stenosis) is superior $(P \leq$ .043) when contrast enhancement gradients are used to estimate the distribution of blood flow to each coronary artery branch (area under the receiver operating characteristic curve $[\mathrm{AUC}]=0.95)$ compared with estimating the distribution of flow on the basis of coronary artery anatomy alone (AUC $\leq$ 0.88).

- FFR can be estimated from CT angiography for lesions of intermediate stenosis severity by using four distinct steps, namely: (a) coronary segmentation, (b) estimation of the myocardial blood flow demand by using myocardial mass, (c) determination of the distribution of the blood flow to each coronary artery branch by using either coronary anatomy or the transluminal attenuation gradient, and (d) application of a computational fluid dynamic simulation.

- A CT FFR algorithm whose details are fully described and that can be performed on site by using a standard desktop computer within 1 hour has been validated against invasive FFR measurements for stenoses of intermediate severity.
Estimation of FFR from CT involves computationally solving for the pressure drop across a coronary artery, given the blood flow required to supply the myocardium subtended by that artery at stress (4). Conceptually, this involves the following four steps: (a) coronary lumen segmentation and (b) estimation of total blood flow through the coronary tree at maximum hyperemia in conjunction with (c) determination of the distribution of this blood flow to each individual branch of the coronary tree and (d) CFD simulation of the blood flow under the so-called boundary conditions a through $c$ to solve for pressure. There is no currently accepted "gold standard" for performing any of these steps. Our general hypothesis was that optimizing the methods used to accomplish each step may help increase CT FFR diagnostic accuracy. The purpose of this work was to compare the diagnostic accuracy of different CT FFR algorithms for vessels with intermediate stenosis.

\section{Materials and Methods}

\section{Study Population}

This cross-sectional retrospective study was Health Insurance Portability and Accountability Act compliant and was approved by the human research committees of all institutions involved. The human research committees waived the requirement for written informed consent on the basis that this was a records-review-only study that posed no more than minimal risk to subjects, that the waiver would not adversely affect the rights and welfare of the

\section{Implication for Patient Care}

- FFR estimated from CT angiography by using a four-step CT FFR algorithm on site in clinically viable times with or without a proprietary basis is superior to coronary CT angiography alone in revealing lesions of intermediate stenosis severity that are hemodynamically significant (FFR $\leq 0.8)$. subjects, that the research could not practicably be performed without the waiver, and that, whenever appropriate, the subjects would be provided with additional pertinent information after participation. No additional imaging or procedures were performed for this study, and no results from this study were used for patient care.

Eligible participants were identified by querying the medical records in one large referral center. CT angiography at that center is performed following established criteria, generally in symptomatic patients with low-to-intermediate risk for coronary artery disease. Clinical decisions to perform invasive conventional coronary angiography (CCA) and FFR are also based on applicable guidelines (9), including the presence or absence of coronary CT angiography findings, as well as other noninvasive functional test results, symptoms, response to therapy, and other etiologic considerations (eg, concern for alterations in coronary motility and vasospasm).

\section{https://doi.org/10.1148/radiol.2017162620 \\ Content codes: CA CT \\ Radiology 2018; 287:76-84 \\ Abbreviations: \\ $\mathrm{AUC}=$ area under the ROC curve \\ CCA = conventional coronary angiography \\ $\mathrm{CFD}=$ computational fluid dynamic \\ $\mathrm{DS}=$ diameter stenosis \\ $\mathrm{FFR}=$ fractional flow reserve \\ $\mathrm{ROC}=$ receiver operating characteristic \\ SCCT $=$ Society of Cardiovascular Computed Tomography \\ STL = Standard Tesselation Language \\ TAG $=$ transluminal attenuation gradient}

\section{Author contributions:}

Guarantors of integrity of entire study, S.K., N.K., K.T., J.A.C.L., F.J.R., D.M.; study concepts/study design or data acquisition or data analysis/interpretation, all authors; manuscript drafting or manuscript revision for important intellectual content, all authors; manuscript final version approval, all authors; agrees to ensure any questions related to the work are appropriately resolved, all authors; literature research, S.K., A.A.G., A.T., Y.S.C., J.A.C.L., F.J.R. D.M.; clinical studies, S.K., N.K., Y.H., K.T., J.A.C.L., F.J.R.; experimental studies, S.K., A.A.G., A.T., Y.H., F.J.R., D.M.; statistical analysis, A.A.G., A.T., D.M.; and manuscript editing, S.K., A.A.G., Y.S.C., C.D., Y.H., J.A.C.L., F.J.R., D.M.

Conflicts of interest are listed at the end of this article. See also the editorial by Leipsic and Koweek in this issue 
Records search criteria were patients who had undergone a CT angiography examination between June 2012 and May 2015 and who subsequently underwent CCA within 90 days. Among the 799 patients identified, we excluded all patients who did not have a pressure measurement in an unstented coronary artery with a lesion of intermediate stenosis severity as determined at CT angiography, which we defined as $25 \%-69 \%$ diameter stenosis (DS) based on Society of Cardiovascular Computed Tomography (SCCT) recommendations (10). This excluded 714 patients. Of the 85 remaining patients, 22 had only an instantaneous wave-free ratio, or iFR, measurement (11) with no FFR measurement in the target vessel, and these patients were also excluded. Among the 63 remaining patients, we additionally sought to exclude patients with a cardiac event between CT angiography and FFR $(n=$ $0)$, myocardial infarction fewer than 30 days prior to CT angiography $(n=0)$, and prior coronary artery bypass graft placement $(n=0)$. The final cohort identified was thus 63 subjects. Two patients were excluded from analysis owing to failure of the semiautomated software used for coronary segmentation. Sixty-one patients were thus included in the study. In those patients, FFR was measured, on average, 36.5 days after CT angiography (median, 24 days; interquartile range, 14-39 days).

CT angiography.-Patients were imaged axially with a first-generation $320 \times 0.5-\mathrm{mm}$ detector row CT scanner (Aquilion ONE; Toshiba Medical Systems, Otawara, Japan) (12). Patients with a heart rate of 65 or more beats per minute received 20 $\mathrm{mg}$ of oral metoprolol 3 hours before imaging. For patients whose heart rate remained at 65 or more beats per minute, $0.125 \mathrm{mg}$ intravenous landiolol (Ono Pharmaceutical, Osaka, Japan) per kilogram of body weight was administered immediately prior to imaging. The average heart rate during CT angiography was 64 beats per minute \pm 9 (range, 46-88 beats per minute). Patients with systolic blood pressure of $110 \mathrm{~mm} \mathrm{Hg}$ or greater received $0.3 \mathrm{mg}$ sublingual nitroglycerin. Contrast material (4-5 $\mathrm{mL}$ iopamidol [370 milligrams of iodine per milliliter]; Bayer Healthcare, Osaka, Japan) followed by saline (20 $\mathrm{mL}$ ) were injected intravenously with a power injector at a rate of $4-5 \mathrm{~mL} /$ sec. Data were acquired at $120 \mathrm{kV}$ with tube current modulation (300$400 \mathrm{~mA}$ ), timed by bolus tracking in the ascending aorta by using a 200HU threshold. Images were reconstructed at $0.5-\mathrm{mm}$ thickness with $0.25-\mathrm{mm}$ overlap by using filtered back projection. A board-certified cardiologist with SCCT Level 3 cardiac CT certification and 10 years of experience in cardiac imaging (S.K.) retrospectively analyzed the CT angiography images to measure percentage DS of the target lesion using quantitative coronary CT angiography software (QAngio CT; MEDIS, Leiden, the Netherlands). A lesion with a percentage DS of $50 \%$ or greater at quantitative coronary CT angiography was considered obstructive.

CCA and FFR measurement.CCA and FFR were performed according to the institutional clinical protocol $(13,14)$ with a femoral or radial approach. Briefly, FFR was measured by using a pressure wire (Verrata Pressure Guide Wire, VOLCANO or Pressure Wire Aeris, St Jude Medical) advanced past the stenosis after intracoronary injection of papaverine hydrochloride (left coronary artery: $12 \mathrm{mg}$; right coronary artery: $8 \mathrm{mg}$ ). The measurement was repeated if FFR drift was greater than 0.03. An FFR of 0.80 or less was considered hemodynamically significant $(9,11)$. CCA images were retrospectively analyzed (S.K.) by using quantitative coronary angiography software (QAngio; MEDIS) to determine percentage DS of the target lesion (15).

\section{CT FFR Algorithm}

Coronary segmentation.-Coronary segmentation was performed by using commercial segmentation software (Toshiba Cardiac Analysis Package, Toshiba Medical Systems). Manual editing was performed as necessary by a cardiology fellow with 1 year of experience in cardiac imaging (A.A.G.). Segmented lumen contours were output by the segmentation software at $0.5-\mathrm{mm}$ intervals along the vessel centerlines to reflect the largest voxel dimension of the CT angiography data (section thickness) and were combined to yield a Standard Tesselation Language (STL) file of the endoluminal surface for further CFD analysis (details provided in Appendix E1 [online]).

Hyperemic blood flow demand calculations.-Total resting myocardial blood flow was calculated assuming myocardium requires $0.8 \mathrm{~mL} / \mathrm{min} / \mathrm{g}$ of blood at rest (16). The left ventricular myocardium, assumed to represent two-thirds of total myocardial mass (17), was automatically segmented from CT angiography images in Vitrea 6.7 (Vital Images, Minnetonka, Minn) by a 2nd-year medical student with 6 months of experience in coronary CT angiography postprocessing (A.T.). To estimate hyperemic flow, we assumed that epicardial coronary arteries presented negligible resistance to flow and that total distal resistance was reduced to one-quarter its resting-state value $(16,18)$.

Blood flow distribution calculations. - Conservation of flow was used to calculate the distribution of flow to each coronary branch. Specifically, flow arriving at a bifurcation was conserved in the two daughter branches. Three models were used to determine the relative flow to each branch. The first two assumed that flow was proportional to the coronary diameter to one of two powers, either the third power $\left(\mathrm{Q} \sim \mathrm{D}^{3}\right.$, the Murray law [19]) or the seven-thirds power $\left(\mathrm{Q} \sim \mathrm{D}^{7 / 3}\right.$, the Huo-Kassab model [20]). To apply these methods, the diameter of each branch was measured (by A.A.G.) immediately after, or at the first nondiseased location after, the bifurcation in cross-sectional images, orthogonal to the vessel centerline. The fraction of flow proceeding to each daughter branch was then calculated by solving the resulting equations (eg, for a bifurcation using the Murray law, $Q_{\text {parent }}=Q_{\text {branch1 }}+Q_{\text {branch2 }}$, $\mathrm{Q}_{\text {branch1 }} \sim \mathrm{D}_{\text {branch1 }}{ }^{3}$ and $\left.\mathrm{Q}_{\text {branch2 } 2} \sim \mathrm{D}_{\text {branch2 }}{ }^{3}\right)$. The third model used the coronary contrast 
opacification gradient (transluminal attenuation gradient [TAG]) (21) to estimate flow. Specifically, we related the TAG to blood flow in a coronary artery as Q -1/TAG (Appendix E1 [online]) and calculated the relative flow to each branch identically to the previous two models. TAG was measured (A.A.G.) by using previously validated software (22). The measurement was performed from the coronary ostia to a distal location where the vessel lumen cross-sectional area tapered to approximately the same size for each daughter branch (1.5-2.0 $\mathrm{mm}^{2}$ ) to minimize differences in luminal opacification measurements due to differing vessel calibers (23).

CFD simulations. - A commercial CFD software suite (ANSYS, Canonsburg, Pa) was used to solve the incompressible Navier-Stokes equations at steady state by using previously optimized procedures $(24,25)$ (details provided in Appendix E1 [online]). The CFD solver was applied (by A.T.) identically for each of the three blood flow distribution models.

CT FFR calculation.-The CT FFR algorithm steps described above were performed on standard desktop workstations (Hewlett Packard, Intel Xeonbased; $4 \times 2.67 \mathrm{GHz}$ with $48 \mathrm{~GB}$ RAM for CFD, and $4 \times 3.00 \mathrm{GHz}$ with $16 \mathrm{~GB}$ RAM for segmentation) with blinding to all patient characteristics, including CT angiography, CCA, and FFR findings. The workflow is shown in Figure E1 (online). For the final 11 patients, when sufficient workflow proficiency was established, the time required to perform each step was recorded. To calculate CT FFR, the pressure solved by CFD was interrogated at the location matching the invasive FFR measurement. Matching of the location of the FFR pressure wire identified in the CCA images to the CT angiography images was performed (by A.A.G., who was blinded to the FFR measurement). The relative pressure decrease $(\Delta \mathrm{P})$ between the coronary ostium and the site of invasive FFR measurement obtained from the CFD solution for each CT FFR algorithm was used to calculate CT FFR as $P_{\mathrm{d}} / P_{\mathrm{a}}$, where $P_{\mathrm{d}}=$ $P-\Delta \mathrm{P}$, and the mean aortic pressure was estimated as $P_{\mathrm{a}}=1 / 3 \cdot \mathrm{sBP}+2 / 3$.

\begin{tabular}{|c|c|}
\hline \multicolumn{2}{|c|}{ Study Population Characteristics $(n=61)$} \\
\hline Characteristic & Datum \\
\hline \multicolumn{2}{|l|}{ Demographic data } \\
\hline No. of male patients & $51(84)$ \\
\hline Age $(y)^{\star}$ & $69.3 \pm 10.1$ \\
\hline Height $(\mathrm{cm})^{\star}$ & $163.1 \pm 9.0$ \\
\hline Weight $(\mathrm{kg})^{\star}$ & $66.9 \pm 15.6$ \\
\hline Body mass index $\left(\mathrm{kg} / \mathrm{cm}^{2}\right)^{\star}$ & $25.0 \pm 4.2$ \\
\hline \multicolumn{2}{|l|}{ CAD risk factor } \\
\hline Diabetes mellitus & $31(51)$ \\
\hline Hypertension & $47(77)$ \\
\hline Hyperlipidemia & $45(74)$ \\
\hline Smoking & $16(26)$ \\
\hline \multicolumn{2}{|l|}{ CAD history } \\
\hline Prior myocardial infarction & $5(8)$ \\
\hline $\begin{array}{l}\text { Prior percutaneous coronary } \\
\text { intervention }\end{array}$ & $6(10)$ \\
\hline \multicolumn{2}{|l|}{ Invasive FFR measurements } \\
\hline LAD artery & $46(75)$ \\
\hline LCx artery & $6(10)$ \\
\hline RCA artery & $9(15)$ \\
\hline CAD in target vessels & 61 \\
\hline DS at quantitative CT angiography (\%) ${ }^{*}$ & $46.8 \pm 8.6$ \\
\hline Mild (25\%-49\% DS) & $38(62)$ \\
\hline Moderate $(50 \%-69 \%$ DS) & $23(38)$ \\
\hline DS at quantitative angiography $(\%)^{*}$ & $47.2 \pm 8.6$ \\
\hline Nonobstructive (<50\% DS) & $37(61)$ \\
\hline Obstructive ( $\geq 50 \%$ DS) & $24(39)$ \\
\hline
\end{tabular}

Note.- Unless otherwise specified, data are numbers of patients, with percentages in parentheses. $C A D=$ coronary artery disease, $\mathrm{LAD}=$ left anterior descending, $\mathrm{LCx}=$ left circumflex, $\mathrm{RCA}=$ right coronary artery.

* Data are means \pm standard deviations.

dBP from systolic (sBP) and diastolic (dBP) pressure measurements obtained at the time of CT angiography.

\section{Statistical Analysis}

Statistical analyses were performed in STATA 9.2 (STATA, Tex). We first calculated Pearson $(r)$ and Spearman ( $\rho)$ correlation coefficients and BlandAltman bias and limits of agreement between each CT FFR algorithm and invasive FFR. Second, we calculated the area under the receiver operating characteristic (ROC) curve (AUC) of each CT FFR algorithm and of $50 \%$ or greater DS at CT angiography for revealing an invasive FFR of 0.8 or less. The AUCs were compared by using the DeLong method. $P<.05$ was considered to indicate a statistically significant difference.

\section{Results}

Patient characteristics are provided in Table 1. No patient had more than one invasive FFR measurement in a vessel with intermediate stenosis severity (25\%-69\%) at quantitative coronary CT angiography. The average target lesion percentage DS was $47.2 \% \pm 8.6$ (range, $30.9 \%-64.8 \%$ ) at quantitative coronary CT angiography. Twenty-five lesions (41\%) had an FFR of 0.8 or less.

The AUC of CT FFR using TAG $(\mathrm{AUC}=0.953)$ was significantly higher than that using the Huo-Kassab rule $(\mathrm{AUC}=0.882)$ or Murray law $(\mathrm{AUC}=$ $0.871)$ models $(P=.043$ and $P=.033$, respectively; Fig 1$)$. There was no significant difference in AUC between the Murray law and Huo-Kassab CT FFR algorithms $(P=.286)$. Average 


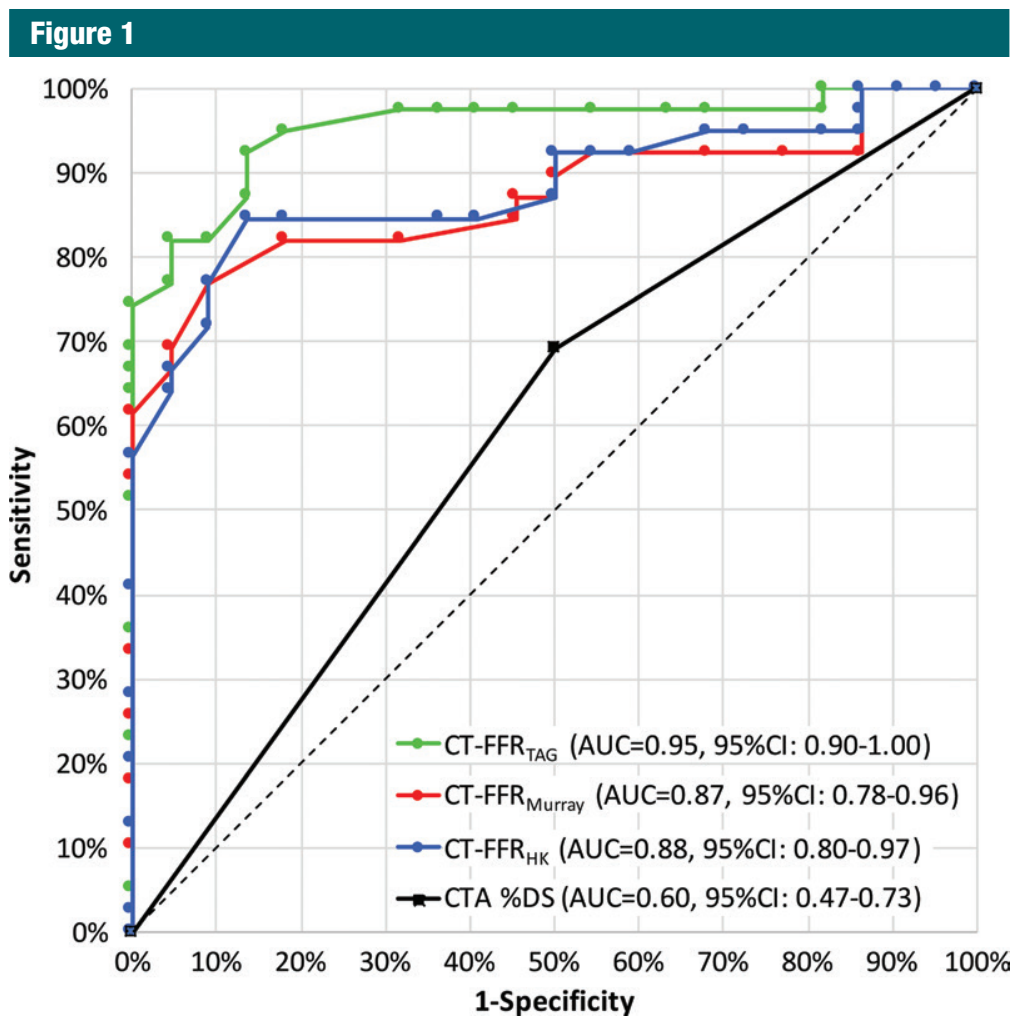

Figure 1: Graph shows ROC curve of CT FFR for the detection of invasive FFR of 0.8 or lower by using each of three models (TAG, Murray law, and Huo-Kassab [HK] rule) to determine the relative distribution of blood flow to each coronary artery.

per-patient CT FFR analysis time was 59.4 minutes \pm 16.0 , including vessel tracing (10.4 minutes \pm 2.7$)$, segmentation (16.8 minutes \pm 3.8$)$, measuring parameters for flow distribution boundary condition calculations (6.0 minutes \pm 2.3 ), generating the finite volume mesh for CFD calculations (13.4 minutes \pm 2.9 ), and performing the CFD (12.8 minutes \pm 5.4). Discrimination of hemodynamically significant disease was improved with all three CT FFR algorithms compared with DS of $50 \%$ or greater at CT angiography (AUC = 0.596; $P<.001$ for all). Point estimates of accuracy, sensitivity, and specificity of each CT FFR algorithm and CT angiography to reveal FFRs of 0.8 or less are provided in Table 2.

Correlation of CT FFR and invasive FFR was good for all three CT FFR models. It was highest for TAG, followed by the Huo-Kassab rule and Murray law CT FFR estimates (Table 2). All three models yielded a low bias (Fig 2). Bland-Altman limits of agreement were narrowest for the TAG model, followed by the Huo-Kassab and Murray law models (Fig 2, Table 2). Two cases, one in which there was agreement of CT FFR estimates between the three flow distribution models, and one in which there was disagreement, are shown in Figure 3.

\section{Discussion}

The main findings of our study are that (a) CT FFR measurement performed on site by using a four-step algorithm implemented without a proprietary basis improves identification of hemodynamically significant intermediate lesions compared with coronary CT angiography and (b) CT FFR accuracy for those lesions is increased when contrast opacification gradients are used to determine the patient-specific distribution of blood flow among coronary arteries rather than using coronary anatomy for this purpose.

Lesions of intermediate stenosis severity pose a challenge for management, as they can cause ischemia despite not appearing angiographically severe. A study of patients with chest pain (26) in stable condition referred for coronary angiography reported rates of invasive FFR of 0.8 or less of $13 \%$ for lesions of less than $30 \%$ DS, $33 \%$ for lesions of $31 \%-50 \%$ DS, and $33 \%$ for lesions of $50 \%-70 \%$ DS. Current guidelines document the utility of FFR to determine if percutaneous coronary intervention is beneficial in "intermediate" or "indeterminant” lesions (27). CT FFR algorithms can estimate FFR from CT angiography data toward reducing unnecessary invasive angiography referrals (2). The accuracy of several such algorithms has generally been favorable (3,5-8), including for the important minority of patients with stenoses of intermediate severity $(5,7,28,29)$ (Table E1 [online]).

Using the initial CT FFR technology (FFR ${ }_{\mathrm{ct}}$, Heartflow), Min et al (28) first reported an accuracy of $86 \%$ for the detection of FFR of 0.8 or less in lesions with 40\%-70\% angiographic stenosis. A subsequent study of that technology (29) found a lower accuracy of $71 \%$ for lesions with $30 \%-69 \%$ stenosis at CT angiography, but a nonetheless improved discrimination of hemodynamically significant disease compared with CT angiography interpretation alone. Coenen et al (7) reported a similar accuracy of $71.5 \%$ in lesions with $25 \%-69 \%$ stenosis at CT angiography using a different CT FFR algorithm (cFFR, Siemens Healthcare). The most recent NXT trial of the initial $\mathrm{FFR}_{\mathrm{ct}}$ technology improved on these results, realizing an accuracy of $80 \%$ in vessels with $30 \%-70 \%$ stenosis at CT angiography (5). The authors of that study attributed the improved performance in part to the use of a "substantially refined” $\mathrm{FFR}_{\mathrm{ct}}$ algorithm. To estimate invasive FFR, CT FFR algorithms must simulate the blood flow through each coronary artery at hyperemia from CT angiography data acquired at rest. The model used to estimate this flow can affect CT FFR accuracy. For 


\section{Table 2}

\begin{tabular}{|c|c|c|c|c|c|c|c|}
\hline $\begin{array}{l}\text { Modality and } \\
\text { Measurement }\end{array}$ & AUC & Sensitivity & Specificity & Accuracy & $\begin{array}{l}\text { Correlation } \\
\text { (Spearman } \rho \text {, } \\
\text { Pearson } \eta \text { ) }\end{array}$ & Bias & $\begin{array}{l}\text { Limits of } \\
\text { Agreement }\end{array}$ \\
\hline $\begin{array}{c}\text { CT angiography } \\
\geq 50 \% \text { DS }\end{array}$ & $0.596(0.467,0.726)$ & $0.500(0.319,0.671)$ & $0.692(0.590,0.789)$ & $0.623(0.492,0.746)$ & $\rho=-0.24, r=-0.27$ & NA & NA \\
\hline CT FFR ${ }_{\text {Murray }}$ & $0.871(0.782,0.960)$ & $0.909(0.739,0.983)$ & $0.821(0.724,0.862)$ & $0.852(0.730,0.906)$ & $\rho=0.67, r=0.52$ & $-0.015(-0.049,0.020)$ & $-0.285,0.256$ \\
\hline CT FFR ${ }_{\mathrm{HK}}$ & $0.882(0.798,0.967)$ & $0.864(0.690,0.960)$ & $0.846(0.748,0.900)$ & $0.852(0.727,0.922)$ & $\rho=0.68, r=0.56$ & $-0.049(-0.074,0.024)$ & $-0.246,0.149$ \\
\hline CT FFR $\mathrm{TAG}_{\mathrm{T}}$ & $0.953(0.902,1.000)$ & $0.949(0.859,0.990)$ & $0.864(0.705,0.936)$ & $0.918(0.804,0.970)$ & $\rho=0.80, r=0.73$ & $-0.018(-0.039,0.004)$ & $-0.182,0.147$ \\
\hline
\end{tabular}

Note. - The Youden index for three models was used to determine the relative distribution of blood flow to each coronary artery. HK = Huo-Kassab, NA = not applicable. Data in parentheses are $95 \%$ confidence intervals.

\section{Figure 2}

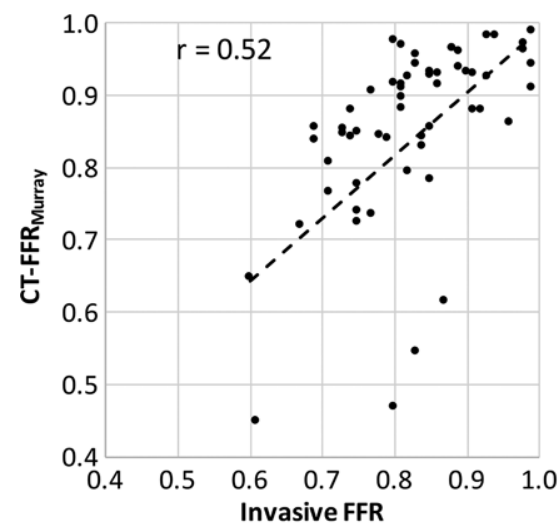

a.

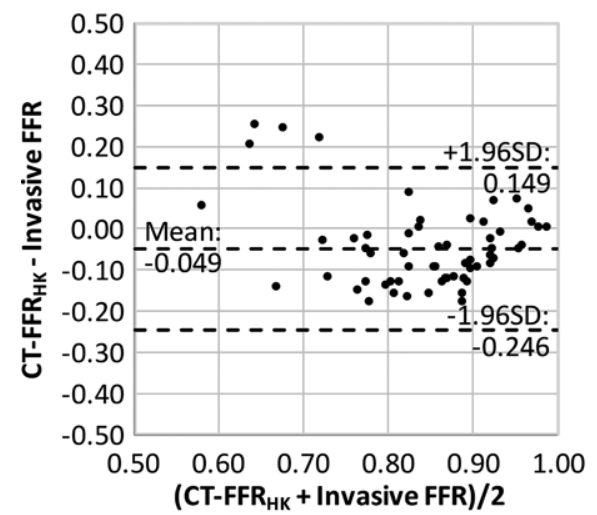

d.

Figure 2: $\quad(a, c, e)$ Correlation and $(b, d, f)$ Bland-Altman plots of invasive FFR measurements and CT FFR estimates for each of three models to determine the relative distribution of blood flow to each coronary artery: Murray law $(\mathbf{a}, \mathbf{b})$, Huo-Kassab $(H K)$ rule $(\mathbf{c}, \mathbf{d})$, and TAG $(\mathbf{e}, \mathbf{f}) . S D=$ standard deviation.

example, Ko et al (6) failed to find a statistically significant improvement in detecting hemodynamically significant lesions compared with CT angiography

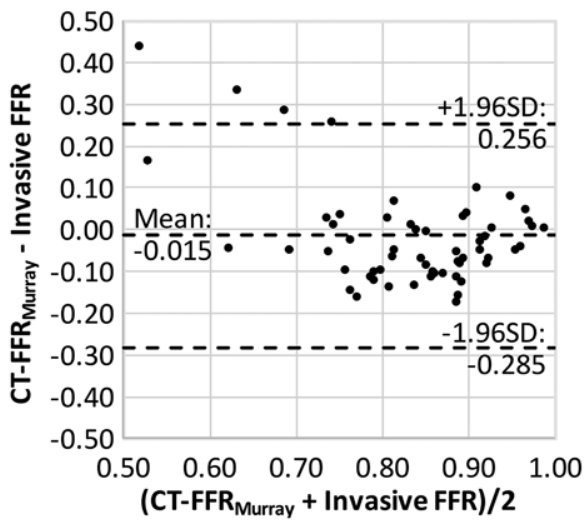

b.
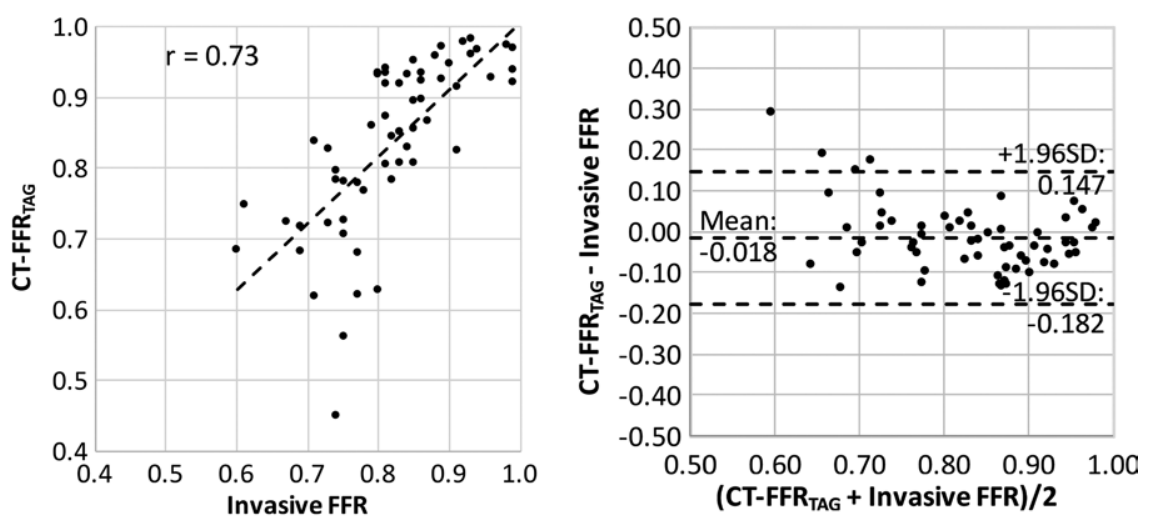

f.

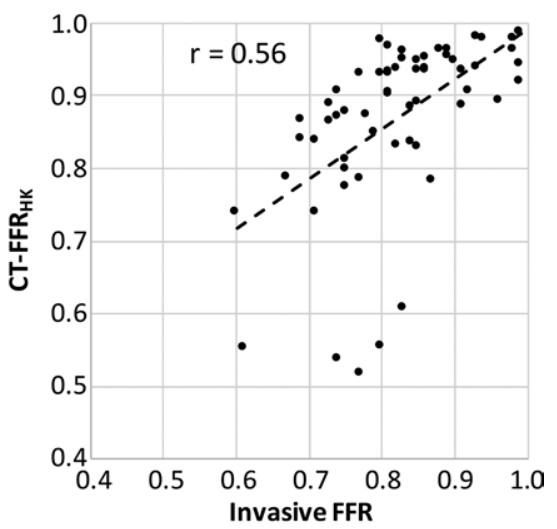

c. 


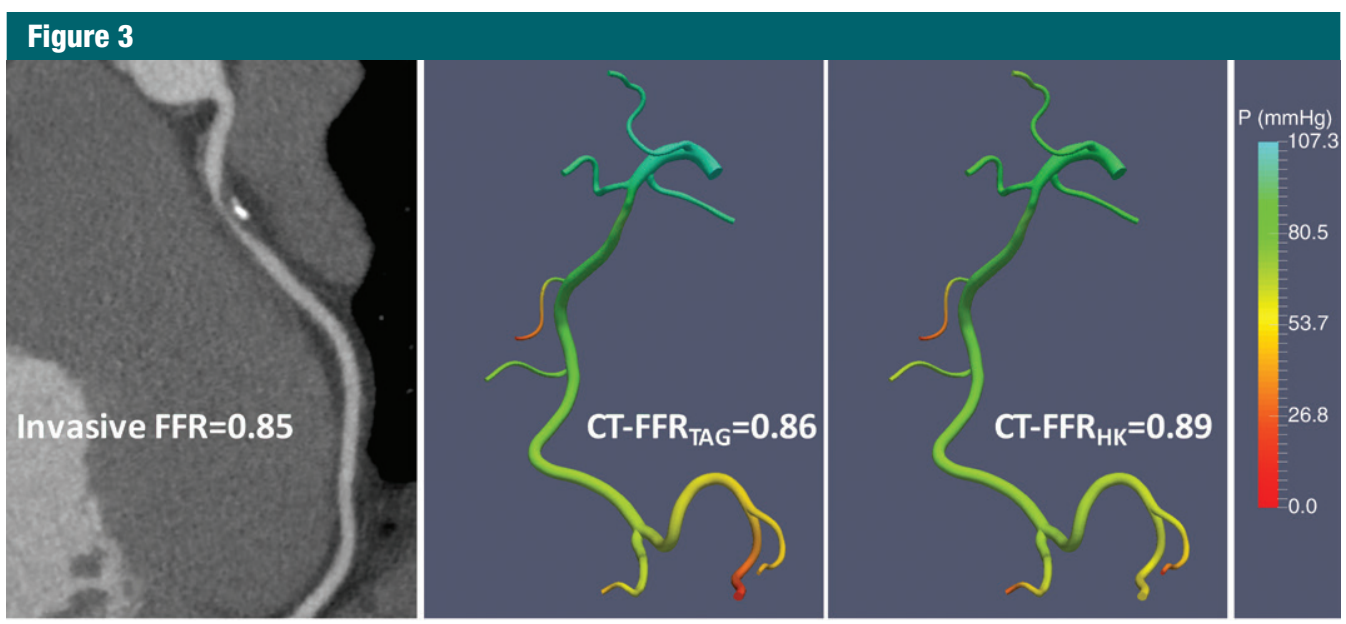

a.
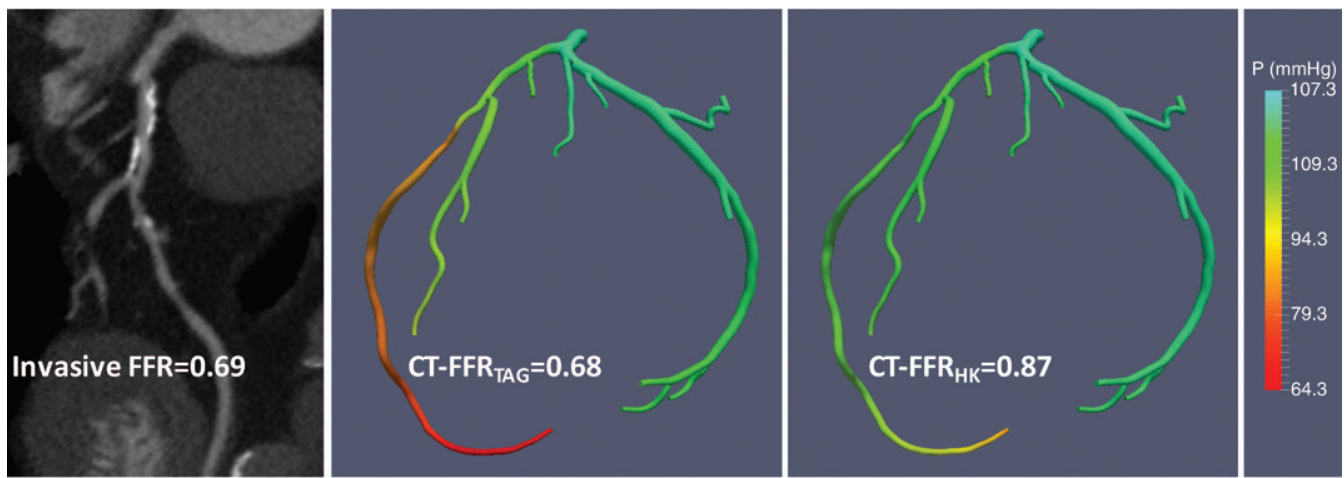

b.

Figure 3: CT angiography and CT FFR estimates for two vessels included in this study. (a) The CT FFR estimate for a lesion in a right coronary artery, wherein vessel diameter is easy to measure, is concordant with the invasive FFR measurement of greater than 0.8 using either a coronary anatomy-based model (Huo-Kassab [HK] rule shown) or the TAG for CT FFR calculations. (b) In contrast, in a left anterior descending coronary artery with a long lesion extending across two bifurcations, wherein measurement of coronary diameter is difficult, the CT FFR estimate based on coronary anatomy is discordant with the invasive FFR measurement of 0.8 or lower, while that based on the TAG is in agreement.

that were comparable to that in the study by Min et al (28). However, correlation and limits of agreement for invasive FFR were lower, likely resulting in a lower AUC than in that study and closer to that in the study by Nakazato et al (29). Coronary contrast opacification gradients are correlated with Thrombolysis in Myocardial Infarction (TIMI) flow grade (30) and TIMI frame counts (31), suggesting they contain information regarding coronary flow that may in turn be useful for improving CT FFR calculations. This may explain the improved correlation and limits of agreement for invasive FFR and the higher overall accuracy $(92 \%)$ of a CT FFR algorithm using contrast material gradients compared with results in prior studies $(5,7,28,29)$. Importantly, all three CT FFR algorithms improved the identification of hemodynamically significant lesions compared with CT anatomic severity assessment, in agreement with the findings of the majority of CT FFR studies.

The results of this study should be carefully interpreted considering its retrospective, single-center design. Our findings do not generalize to more severe lesions that were not studied. Our results may also not generalize to lowerrisk populations typically referred to CT angiography, because the decision to measure FFR in the lesions studied was clinically motivated, introducing selection bias toward angiographically borderline obstructive disease and/or other patient characteristics and test results suggestive of ischemia. This bias is reflected in the significant proportion of hemodynamically significant lesions in our study, which was similar to that in other comparable studies (7). The Murray law and Huo-Kassab CT FFR algorithms can be applied regardless of the number of CT detector rows used for CT angiography data acquisition. However, relating coronary flow to coronary opacification gradients in theory requires isotemporal CT angiography acquisition (12), although corrections 
for CT with fewer than 256 rows may be possible (30). Lesions with percentage DS greater than $80 \%$ affect resting flow (32), which may both affect the use of contrast opacification gradients for CT FFR calculations and violate the assumption of minimal epicardial coronary resistance to flow. Modifications to the algorithm presented here would be required to address these limitations. Other assumptions that were used to determine myocardial blood flow requirements may not hold for certain patients, such as those with left ventricular hypertrophy (relationship of flow to myocardial mass) or diabetes mellitus (response of microvascular resistance to the administration of adenosine) (33). On the basis of positive results in a challenging subset of coronary lesions, we expect that further modifications and optimizations of the methods underlying CT FFR computations may improve its availability and performance beyond the current state of the art.

Disclosures of Conflicts of Interest: S.K. disclosed no relevant relationships. A.A.G. disclosed no relevant relationships. A.T. disclosed no relevant relationships. N.K. disclosed no relevant relationships. Y.S.C. disclosed no relevant relationships. C.D. disclosed no relevant relationships. Y.H. disclosed no relevant relationships. K.T. Activities related to the present article: received honorarium for lecture from Toshiba. Activities not related to the present article: received honoraria for lectures from Terumo, Kaneka, Zeon, Biosensors, Sanofi, Daiichisankyo, Astra Zeneca Tanabe Mitsubishi, MSD, Otsuka, Novartis, Bristol-Myers, Dainihonsumitomo, Pfizer, Toaeiyo, Nihon Stent Technology, Teijin Pharma, Goodman, Mochida, Eisai, Edwards, St Jude Medical, and Abbott; is on the advisory board of Abbott. Other relationships: disclosed no relevant relationships. J.A.C.L. Activities related to the present article: disclosed no relevant relationships. Activities not related to the present article: has received grant support from Toshiba Medical Systems. Other relationships: disclosed no relevant relationships. F.J.R. disclosed no relevant relationships. D.M. disclosed no relevant relationships.

\section{References}

1. Tonino PA, De Bruyne B, Pijls NH, et al. Fractional flow reserve versus angiography for guiding percutaneous coronary intervention. N Engl J Med 2009;360(3):213-224.

2. Hlatky MA, De Bruyne B, Pontone G, et al. Quality-of-Life and Economic Outcomes of Assessing Fractional Flow Reserve With Com- puted Tomography Angiography: PLATFORM. J Am Coll Cardiol 2015;66(21):2315-2323.

3. Min JK, Leipsic J, Pencina MJ, et al. Diagnostic accuracy of fractional flow reserve from anatomic CT angiography. JAMA 2012;308(12):1237-1245.

4. Taylor CA, Fonte TA, Min JK. Computational fluid dynamics applied to cardiac computed tomography for noninvasive quantification of fractional flow reserve: scientific basis. J Am Coll Cardiol 2013;61(22):2233-2241.

5. Nørgaard BL, Leipsic J, Gaur S, et al. Diagnostic performance of noninvasive fractional flow reserve derived from coronary computed tomography angiography in suspected coronary artery disease: the NXT trial (Analysis of Coronary Blood Flow Using CT Angiography: Next Steps). J Am Coll Cardiol 2014;63(12):1145-1155.

6. Ko BS, Cameron JD, Munnur RK, et al. Noninvasive CT-derived FFR based on structural and fluid analysis: a comparison with invasive FFR for detection of functionally significant stenosis. JACC Cardiovasc Imaging 2017;10(6):663-673.

7. Coenen A, Lubbers MM, Kurata A, et al. Fractional flow reserve computed from noninvasive CT angiography data: diagnostic performance of an on-site clinician-operated computational fluid dynamics algorithm. Radiology 2015;274(3):674-683.

8. Koo BK, Erglis A, Doh JH, et al. Diagnosis of ischemia-causing coronary stenoses by noninvasive fractional flow reserve computed from coronary computed tomographic angiograms: results from the prospective multicenter DISCOVER-FLOW (Diagnosis of Ischemia-Causing Stenoses Obtained Via Noninvasive Fractional Flow Reserve) study. J Am Coll Cardiol 2011;58(19):1989-1997.

9. Patel MR, Bailey SR, Bonow RO, et al. ACCF/ SCAI/AATS/AHA/ASE/ASNC/HFSA/HRS/ SCCM/SCCT/SCMR/STS 2012 appropriate use criteria for diagnostic catheterization: a report of the American College of Cardiology Foundation Appropriate Use Criteria Task Force, Society for Cardiovascular Angiography and Interventions, American Association for Thoracic Surgery, American Heart Association, American Society of Echocardiography, American Society of Nuclear Cardiology, Heart Failure Society of America, Heart Rhythm Society, Society of Critical Care Medicine, Society of Cardiovascular Computed Tomography, Society for Cardiovascular Magnetic Resonance, and Society of Thoracic Surgeons. J Am Coll Cardiol 2012;59(22):19952027.

10. Leipsic J, Abbara S, Achenbach S, et al. SCCT guidelines for the interpretation and reporting of coronary CT angiography: a report of the
Society of Cardiovascular Computed Tomography Guidelines Committee. J Cardiovasc Comput Tomogr 2014;8(5):342-358.

11. Davies JE, Sen S, Dehbi HM, et al. Use of the instantaneous wave-free ratio or fractional flow reserve in PCI. N Engl $\mathrm{J}$ Med 2017;376(19):1824-1834.

12. Rybicki FJ, Otero HJ, Steigner ML, et al. Initial evaluation of coronary images from 320-detector row computed tomography. Int J Cardiovasc Imaging 2008;24(5):535-546.

13. Lotfi A, Jeremias A, Fearon WF, et al. Expert consensus statement on the use of fractional flow reserve, intravascular ultrasound, and optical coherence tomography: a consensus statement of the Society of Cardiovascular Angiography and Interventions. Catheter Cardiovasc Interv 2014;83(4):509-518.

14. Naidu SS, Rao SV, Blankenship J, et al. Clinical expert consensus statement on best practices in the cardiac catheterization laboratory: Society for Cardiovascular Angiography and Interventions. Catheter Cardiovasc Interv 2012;80(3):456-464.

15. Kato N, Kishi S, Arbab-Zadeh A, et al. Relative atherosclerotic plaque volume by CT coronary angiography trumps conventional stenosis assessment for identifying flow-limiting lesions. Int J Cardiovasc Imaging 2017 Jun 8. [Epub ahead of print]

16. Canty JM Jr. Coronary blood flow and myocardial ischemia. In: Bonow RO, Mann DL, Zipes DP, Libby P, Braunwald E, eds. Braunwald's heart disease, a textbook of cardiovascular medicine. 9th ed. Philadelphia, Pa: Elsevier, 2012; 1049-1075.

17. Ferlinz J. Right ventricular function in adult cardiovascular disease. Prog Cardiovasc Dis 1982;25(3):225-267.

18. Wilson RF, Wyche K, Christensen BV, Zimmer S, Laxson DD. Effects of adenosine on human coronary arterial circulation. Circulation 1990;82(5):1595-1606.

19. Murray CD. The physiological principle of minimum work applied to the angle of branching of arteries. J Gen Physiol 1926;9 (6):835-841 .

20. Huo Y, Kassab GS. Intraspecific scaling laws of vascular trees. J R Soc Interface 2012;9(66): 190-200.

21. Steigner ML, Mitsouras D, Whitmore AG, et al. Iodinated contrast opacification gradients in normal coronary arteries imaged with prospectively ECG-gated single heart beat 320-detector row computed tomography. Circ Cardiovasc Imaging 2010;3(2):179-186.

22. Chatzizisis YS, George E, Cai T, et al. Accuracy and reproducibility of automated, standardized coronary transluminal attenuation 
gradient measurements. Int $\mathrm{J}$ Cardiovasc Imaging 2014;30(6):1181-1189.

23. Paul NS, Blobel J, Kashani H, Rice M, Ursani A. Quantification of arterial plaque and lumen density with MDCT. Med Phys 2010;37(8):4227-4237.

24. Giannopoulos AA, Chatzizisis YS, Maurovich-Horvat P, et al. Quantifying the effect of side branches in endothelial shear stress estimates. Atherosclerosis 2016;251:213-218.

25. Toutouzas K, Chatzizisis YS, Riga M, et al. Accurate and reproducible reconstruction of coronary arteries and endothelial shear stress calculation using 3D OCT: comparative study to 3D IVUS and 3D QCA. Atherosclerosis $2015 ; 240(2): 510-519$.

26. Curzen N, Rana O, Nicholas Z, et al. Does routine pressure wire assessment influence management strategy at coronary angiography for diagnosis of chest pain? the RIPCORD study. Circ Cardiovasc Interv 2014;7(2):248255.
27. Fihn SD, Blankenship JC, Alexander KP, et al. 2014 ACC/AHA/AATS/PCNA/SCAI/STS focused update of the guideline for the diagnosis and management of patients with stable ischemic heart disease: a report of the American College of Cardiology/American Heart Association Task Force on Practice Guidelines, and the American Association for Thoracic Surgery, Preventive Cardiovascular Nurses Association, Society for Cardiovascular Angiography and Interventions, and Society of Thoracic Surgeons. J Am Coll Cardiol 2014;64(18):1929-1949.

28. Min JK, Koo BK, Erglis A, et al. Usefulness of noninvasive fractional flow reserve computed from coronary computed tomographic angiograms for intermediate stenoses confirmed by quantitative coronary angiography. Am J Cardiol 2012;110(7):971-976.

29. Nakazato R, Park HB, Berman DS, et al. Noninvasive fractional flow reserve derived from computed tomography angiography for coronary lesions of intermediate stenosis se- verity: results from the DeFACTO study. Circ Cardiovasc Imaging 2013;6(6):881-889.

30. Chow BJ, Kass M, Gagné O, et al. Can differences in corrected coronary opacification measured with computed tomography pre dict resting coronary artery flow? J Am Coll Cardiol 2011;57(11):1280-1288.

31. Choi JH, Min JK, Labounty TM, et al. Intracoronary transluminal attenuation gradient in coronary CT angiography for determining coronary artery stenosis. JACC Cardiovasc Imaging 2011;4(11):1149-1157.

32. Gould KL. Does coronary flow trump coronary anatomy? JACC Cardiovasc Imaging 2009;2(8):1009-1023.

33. Coenen A, Lubbers MM, Kurata A, et al. Coronary CT angiography derived fractional flow reserve: methodology and evaluation of a point of care algorithm. J Cardiovasc Comput Tomogr 2016;10(2):105-113. 